\title{
Transición del Sistema de Gestión de Calidad: Una revisión de las normas ISO 9001 versión 2008 y 2015
}

\section{Transition of the Quality Management System: A review of ISO 9001 version 2008 and 2015}

Isabel Rios-Dominguez ${ }^{1}$, William Arellano-Cartagena², Cesar Martinez-Pajaro³

${ }^{1}$ Magister en Educación, Facultad de Educación, Grupo de Investigación Educación e Innovación Educativa, Universidad Tecnológica de Bolívar, Calle del Bouquet Cra.21 \#25-92 Barrio Manga, Cartagena de indias, Colombia.

irios@utb.edu.co

2 Director de la Maestría en Educación, Decano de la Facultad de Educación, Director del Grupo de Investigación Educación e Innovación Educativa, Universidad Tecnológica de Bolívar, Calle del Bouquet Cra.21 \#25-92 Barrio Manga, Cartagena de indias, Colombia.

warellano@utb.edu.co

3 Coordinador de la Maestría en Sistemas de Gestión y de la Especialización en Gerencia de la Calidad, Facultad de Ingeniería, Grupo de Investigación CIPTEC, Fundación Universitaria Tecnológico Comfenalco Cartagena, Sede A Barrio España Cr 44 D No 30A - 91, Cartagena de indias, Colombia.

cmartinezp@tecnocomfenalco.edu.co

Recibido: 15/feb/2019 Revisado: 30/abr/2019

Aceptado: 30/may/2019 Publicado: 30/jul/2019

Resumen El siguiente trabajo es una revisión de literatura sobre los sistemas de gestión de calidad, en especial, los sistemas integrados de gestión, que consta de la correspondencia que existe entre las normas ISO 9001: 2015, ISO14001 y OHSAS 1800; esta correspondencia es de tipo técnico y estructural y ayuda a garantizar la calidad en todas las áreas de la empresa de forma clara, directa y acertada. A lo largo del informe se presentan diferentes definiciones que se dan a cada una de las etapas de un sistema integrado de gestión, que se pueden resumir en: introducción a los sistemas integrados de gestión, enfoque basado en procesos y documentación de los sistemas de gestión, responsabilidad de la dirección y gestión de recursos, determinación y revisión de los requisitos, medición, análisis y mejora.

Palabras claves: desarrollo empresarial; partes interesadas; sistema integrado de gestión.

Abstract The following is a review of the literature on quality management systems, especially the integrated management systems, which consists of the correspondence that exists between the ISO 9001: 2015, ISO14001 and OHSAS 18001 standards. This correspondence is of a technical and structural type and it helps to ensure quality in all areas of the company in a clear, direct and accurate way. Throughout the report, There are many different definitions that are given to the characteristics of a management system. They can be summarized as: introduction of integrated management systems, process-based approach and documentation of management systems, management responsibility and resource management, determination and review of requirements, measurement, analysis and improvement.

Keywords Business development; concerned parties; integrated management system. 


\section{Introducción}

En el mundo competitivo de hoy, las organizaciones están llamadas a trabajar eficazmente para encontrar soluciones rápidas y eficientes a los grandes problemas que se le presentan; por ello es preciso que tanto los directivos como los trabajadores, se sientan identificados con esta necesidad y el empleo de modernos Sistemas de

Gestión (SG), lo que la conducirá a la obtención de una mejor posición en el mercado y al cumplimiento de sus objetivos, misión y visión (Damaso, 2010).

Los sistemas de gestión empresarial han experimentado una notable transformación y cambios, en los cuales las exigencias de los clientes-usuariosasociados y consumidores, son las que marcan el ritmo y la dirección de las organizaciones. Una demanda más exigente y especializada, y normativas cada vez más estrictas en el mercado nacional e internacional, son algunos de los factores que han condicionado esta transformación de los sistemas de gestión (Bedoya et al., 2017; González, 2011).

Como consecuencia de todo ello, las empresas se han visto obligadas a adaptarse continuamente para competir en este nuevo mercado, adecuándose a las nuevas exigencias comerciales y los grupos de interesados en la organización (Paulí y Carretero, 2009). Ante estas nuevas exigencias, las empresas se están encontrando con la necesidad de disponer de sistemas de gestión que den mayor confianza a sus clientes actuales y también a los potenciales y que los productos y servicios suministrados cumplan unos requisitos mínimos; además, la utilización de estos sistemas le provee a la empresa mayores niveles de eficiencia y eficacia, lo cual redunda en una mejor posición competitiva.

Durante siglos, las actuaciones a favor de la protección de la salud de los trabajadores se mantienen en valores testimoniales. La revolución industrial del siglo XIX, trae un aumento considerable de los riesgos industriales, de los accidentes y de las enfermedades profesionales (Fraguela, Carral, y Iglesias, 2011). Se producen actuaciones marcadas por un carácter proteccionista y reparador, dirigido a aquellos trabajadores que han sufrido un daño, y especialmente una lesión corporal motivada por un accidente de trabajo. Es la clásica protección de riesgos laborales, con actuaciones fundamentalmente reactivas o posteriores a la producción del daño.

Desde esta perspectiva, la integración de los sistemas de gestión de la calidad, medio ambiente, seguridad industrial y salud ocupacional se presenta como una alternativa válida y necesaria, para que las organizaciones puedan afrontar con éxito los retos que les depara el siglo XXI (Flores, 2013).

El objetivo de cualquier sistema de gestión empresarial, ya sea de calidad, gestión medioambiental o de prevención de riesgos laborales, es proporcionar garantías del cumplimiento de las políticas, de las especificaciones normativas y legislativas, y proceder a la mejora continua de un sistema bien estructurado, así como permitir que este cumplimiento sea demostrable a otras instituciones mediante la documentación y los registros adecuados (Amparo, 2009).

Hoy día, para una excelente gestión empresarial y para la observancia de las exigencias del consumidor, se hace necesario cumplir ciertos requisitos relacionados con estándares de calidad, medio ambiente, salud ocupacional y seguridad industrial; buscando a la vez, incrementar la rentabilidad empresarial (Matiz y Ramírez, 2011).

\section{Introducción a los sistemas integrados de gestión}

Para hablar de un sistema integrado de gestión de la calidad, es importante tener claro la definición de sistema de gestión de la calidad, que de acuerdo a Tejada, (2006) se identifica con la sigla SGC y se define como la forma como una organización realiza la gestión empresarial asociada con la calidad (Beltrán, Carmona, y Carrasco, 2009). En términos generales, consta de la estructura organizacional junto con la documentación, procesos y recursos que se emplean 
para alcanzar los objetivos de calidad y cumplir con los requisitos de los clientes (González, 2008).

El sistema de gestión de calidad contemplado en la norma ISO 9001: 2015 en su versión más reciente, es aquella parte del sistema de gestión de la organización enfocada en el logro de los resultados, en relación con los objetivos de la calidad, para satisfacer las necesidades, expectativas y requisitos de los clientes. Proporciona confianza, tanto interna como externa, sobre la capacidad de la organización para ofrecer productos y servicios que satisfagan los requisitos de forma coherente y es el marco de referencia para la mejora continua con objeto de incrementar la probabilidad de aumentar la satisfacción del cliente (Medina, 2012).

La familia ISO 9000 constituye un conjunto coherente de normas y directrices sobre gestión de la calidad que se han elaborado para asistir a las organizaciones de todo tipo y tamaño, en la implementación y la operación de sistemas de gestión de la calidad (SGC) eficaces (García y Espinel, 2016).

Por su lado, Vidal y Soto, (2013) definen como parte importante el Sistema de Gestión Ambiental, reconocido por la sigla SGA que es parte del sistema de gestión de una organización, empleada para desarrollar e implementar su política ambiental y gestionar sus aspectos ambientales.

En el momento actual, las empresas con visión de futuro saben que la variable ambiental es fundamental para ser competitivos (Morales, 2017). Contrario a lo que se pensaba antes, una actuación ambientalmente amigable es también una herramienta para mejorar la eficiencia productiva de la empresa y obtener beneficios económicos y de mercadeo importantes.

En ese orden de ideas, los Sistemas de Gestión Ambiental son una herramienta pertinente al propósito indicado, pues le permiten a la organización implementar un proceso de manejo ambiental permanente, armónico con el cumplimiento de la legislación y en constante interacción con la comunidad (Pulles y Bataller, 2016).

La norma ISO14001, que define Sistema de Gestión Ambiental como "La parte del Sistema de Gestión general que incluye la estructura organizativa, las actividades de planificación, las responsabilidades, las prácticas, los procedimientos, los procesos y los recursos para desarrollar, implementar, realizar, revisar y mantener la política ambiental (Tejada, 2006).

El Sistema de Gestión Ambiental bajo las normas ISO14001 se encuentra estructurado de la siguiente forma, siguiendo el ciclo PHVA: (i) planear, en esta etapa se definen la política ambiental y la planificación. (ii) hacer, en esta etapa se definen la implementación y operación. (iii) verificar, en esta etapa se lleva la verificación y acción correctiva. (iiii) actuar, en esta etapa se desarrollan las acciones correctivas y la revisión por la dirección (Petro, 2013).

También Fraguela, Carral, e Iglesias, (2011) define el Sistema de Gestión de Seguridad y Salud Ocupacional reconocido con la sigla S\&SO, como parte del sistema de gestión total, que facilita la administración de los riesgos de S\&SO asociados con el negocio de la organización. Incluye la estructura organizacional, actividades de planificación, responsabilidades, prácticas, procedimiento, procesos y recursos, para desarrollar, implementar, cumplir, revisar y mantener la política y objetivos de S\&SO.

Teniéndose en cuenta que la mejora de la productividad y la calidad debe ser estratégica, global e integral, la incidencia de la función de seguridad y salud ocupacional salta a la vista, en particular por el carácter preventivo que debe guiar la gestión de la misma, siendo determinante en muchos casos para obtener los valores esperados de productividad de las organizaciones (Bestraten, 2000). La implantación de un Sistema de Gestión de la Seguridad y Salud en el trabajo, supone una contribución a la mejora en cuanto a condición y factores que afectan al bienestar del entorno físico de una empresa.

La organización que implanta un Sistema de Gestión de seguridad y salud laboral mediante la Norma OHSAS 18001 tiene la garantía de que: cumple con la legislación vigente. Establece un proceso continuo de mejora de su sistema de gestión de la seguridad y salud en el trabajo (Herrera, 2014). Determina y mantiene una capacidad de respuesta ante imprevistos. Facilita la asignación de los recursos en la organización. Busca la mejora continua de la organización, mediante la evaluación de los resultados 
respecto a los objetivos y política anteriormente establecida. Revisa y audita el sistema.

La relación de la normativa de Prevención de Riesgos Laborales con las normas de gestión medioambiental es muy alta y va más allá de sus posibles semejanzas de estructura o planteamientos. Hemos de tener presente que un riesgo laboral se convierte o puede convertirse en un impacto medioambiental dentro de la organización (Benavides, 2013).

Existe una correspondencia técnica entre las normas ISO 9001, ISO 14001 y OHSAS 18001, contemplada en la tabla B1 de la NTC ISO 14001 y en el anexo A de la NTC OHSAS 18001 (Peña, 2011). Además, las normas referenciadas para los sistemas de gestión ambiental y sistema de gestión para seguridad y salud ocupacional, presentan en común el análisis de riesgos ocasionado por los impactos en cualquier medio incluido el humano, que a su vez puede convertirse en un factor de riesgo laboral si éstas personas afectadas son empleados, contratistas o visitantes de la empresa.

También, autores como Rubio, (2002) explica la correspondencia de estructura que existe entre estas normas, así "un sistema integrado de gestión tendría una estructura de árbol, con un tronco común y tres ramas correspondientes a las tres áreas de gestión: calidad, medio ambiente y seguridad y salud laboral" p.175. El tronco tendría en cuenta todos los elementos, desde la política a la asignación de recursos, etc., pasando por la planificación y el control de las actuaciones y terminando con la auditoría y la revisión del Sistema. Cada rama específica de gestión recogería de forma complementaria las cuestiones particulares que la incumben, teniendo siempre en cuenta los aspectos comunes del tronco.

Un Sistema Integral de Gestión SIG es aquel que integra los diversos componentes, procesos y áreas en la consecución de los distintos objetivos y funciones (calidad, salud y seguridad, medio ambiente, personal, finanzas y seguridad, etc.) de un negocio en uno solo (Gisbert y Esenglediev, 2015).

La implantación y certificación de los diversos sistemas, en especial, calidad, salud y seguridad ambiental y ocupacional sistemas (S\&SO) han sido muy importantes para muchas organizaciones debido a la constante demanda creciente de grupos de interés, tanto internos y externos, incluidos los organismos reguladores, sociedad, clientes, empleados, proveedores y las distintas administraciones (Aguilera, 2012).

Así pues, la implantación y mantenimiento de los diversos sistemas y/o normas, conllevan a la realización de cambios y asignación de los recursos existentes que pueden tener un impacto directo en las organizaciones.

Los beneficios de la gestión integrada de los diversos sistemas son: cumplir con todos los requisitos de cada una de las normas, con un conjunto de políticas y procedimientos. Verificar en una sola auditoría los diversos sistemas, ahorrando recursos y reduciendo costes. Mejorar la eficiencia general mediante la eliminación de las tareas duplicadas. Definir funciones y responsabilidades de forma clara frente a objetivos comunes. Que sea más fácil y simple para mejorar continuamente la gestión (Hernández, 2015).

Algunas organizaciones tienen dudas sobre el SIG, cuestionándose con preguntas como ¿Qué valor o valores aportan a la empresa? Otras organizaciones realizan las implementaciones obligadas por los clientes, asociaciones comerciales y otros grupos de interés, no teniendo más remedio que implantar un SIG. Un requisito previo para la integración es un entendimiento de los procesos genéricos y tareas en la gestión del ciclo PHVA (planear-hacer-verificaractuar) que es un método de gestión que se utiliza para el control y la mejora continua de los procesos y productos (Duque, 2010).

Los sistemas integrados de gestión son vistos como una conexión transversal entre las diferentes normas, dado que las mismas tienen una serie de similitudes y actividades comunes (política, planificación, documentación, evaluación, etc.) integración seria, por lo tanto, asegurar que en lugar de dos o más diferentes normas o sistemas, solo exista un estándar en la organización, que podrá ser gestionado y mantenido de manera más eficiente (Cortés, 2014). 
De acuerdo a Luero, (2017) la clave para la verdadera integración de los diferentes sistemas de gestión es sorprendentemente simple: el objetivo del sistema de gestión no debe estar en su aplicación por parte de los diversos gerentes o responsables funcionales, sino más bien que este imbuido en los empleados que realmente producen el producto.

\section{Enfoque basado en procesos y documentación de los Sistemas de Gestión}

Es una plataforma común para unificar los sistemas de gestión de la organización de distintos ámbitos en uno sólo, recogiéndolos en una base documental única. Permite la articulación de los requisitos comunes en un solo sistema. Facilita la gestión, planeación, control y el mejoramiento continuo de las organizaciones (Hernández, Martínez, y Cardona, 2015).

La gestión por procesos, es uno de los conceptos modernos de direccionamiento, que ha resultado como apoyo a la necesidad que tienen las organizaciones de encaminar sus esfuerzos en la construcción de una nueva estructura administrativa (Del prado, 2016). El modelo de gestión basado en procesos, se fundamenta en el cumplimiento de la misión de las organizaciones, direccionando las actividades necesarias, hacia la satisfacción del cliente, proveedores, personal, accionista y sociedad en general. Implantar este tipo de modelo, no solo exige una nueva visión de los procesos, sino que además motiva la generación de valores agregados, para cada una de las variantes que intervienen en todo el modelo. Esta nueva tendencia es lo que diferencia la gestión por procesos de todos los esquemas organizacionales antes conocidos (Pérez, 2010).

Un proceso se define básicamente en su origen, derivado del latín Processus, que significa progreso, avance $o$ adelanto. Bajo estas nuevas orientaciones, los procesos se definen como las diversas actividades que se requieren para generar un resultado, dichas actividades se alimentan de varios componentes (proveedores, insumos, servicios, etc.) que agregan valor al resultado final (Marín, 2013).
Dado que la gestión enfocada a procesos, permite repensar las organizaciones y reorganizar los diferentes subprocesos que la integran, para a través de la mejora continua, permitir nuevas definiciones, controles, seguimientos y formas de operación, se han definido unos objetivos esenciales en esta gestión y son los siguientes:

Conocer las organizaciones de forma sistémica y desarrollar una visión horizontal de la misma.

Favorecer la interacción de equipos de trabajo, para el intercambio de información y la gestión oportuna de soluciones.

Hacer partícipe al recurso humano de todo el modelo, con un mayor sentido de compromiso, en cada uno de los procesos que adelante; indistintamente de su posición jerárquica (López, 2008).

El sistema de gestión basado en procesos surge a raíz de la necesidad de las empresas por integrar las actividades de sus distintas áreas o departamentos en aras de identificar la interrelación de los procesos y de definir las responsabilidades, aspectos claves para garantizar la eficacia de los procesos. De acuerdo con González, (2013) "el enfoque basado en procesos es "un conjunto de actividades mutuamente relacionadas o que interactúan, las cuales transforman elementos de entrada en resultados" ( $p 1$ ), lo cual posibilita a la compañía efectuar un análisis de perfeccionamiento de las funciones y actividades de sus colaboradores para lograr mejoramiento en su competitividad.

El enfoque basado en procesos es de vital importancia para la empresa ya que se impone la necesidad de la gestión por procesos para adaptarse mejor a las necesidades de los clientes y, por tanto, mejorar la competitividad de la organización (Rodríguez, Lezcano, y Varela, 2010).

En lo relacionado a la documentación del sistema integrado de gestión, De Velasco, (2009) afirma que una buena manera de organizar un gestor documental para un sistema integrado de gestión es hacerlo con base a los procesos. Los documentos maestros que hacen parte del sistema son entre otros, la política y los objetivos de gestión y una serie de procedimientos e instructivos que describen los diferentes procesos incluidos en el sistema de gestión (Ponce, 2015). A 
partir de mapa de procesos, dentro de la estructura que alberga cada uno de ellos se asocian los procedimientos, de los cuáles pueden partir formatos o instrucciones técnicas.

Asimismo, se debe tener en cuenta la documentación externa al sistema, como por ejemplo normas que pueden vincularse perfectamente a los procesos (Cárdenas, 2016).

Otro punto de interés es alinear los procesos y/o documentos a los requerimientos de la/s norma/s a los cuáles está dando cumplimiento, por lo que una matriz que enfrente requisitos de la norma frente a la documentación del sistema dan una visualización de gran utilidad. Es muy importante en nuestro sistema de gestión documental:

Que la documentación sea accesible para todo el mundo.

Que las personas tengan accesos controlados a la documentación a través de permisos.

Que no se pueda acceder de forma libre a documentos obsoletos.

Que exista trazabilidad entre las versiones de los documentos.

Que se pueda realizar una distribución rápida y efectiva de nuevas versiones de documentos (Cachay, 2009).

En definitiva, que la documentación trabaje para el sistema de gestión y no el sistema de gestión para la documentación.

\section{El futuro de la documentación en los sistemas de gestión}

El nuevo enfoque de ISO a través del Anexo SL, va a hacer que se deje de hablar de procedimientos y registros y se trabaje con un concepto común de información documentada. En el caso de la ISO 9001:2015 hablará de que la organización debe tener la información requerida por la norma de manera documentada y los documentos determinados por la organización como necesarios para la efectividad de sistema de gestión. Se hace hincapié en decir que el alcance de la información documentada puede diferir de una organización a otra por su tamaño, actividades, procesos, productos y servicios, la complejidad de sus procesos y también de la competencia de las personas (Fernández, 2014).

La norma indicará que al crear y actualizar la información documentada, la organización debe asegurar la identificación y descripción, así como el formato (idioma, versión del software, gráficos), los medios por los cuáles el documento se comunica (papel, formato electrónico, etc.) y la revisión periódica de la misma (Leung, 2010). El sistema de gestión debe asegurar que la documentación esté disponible para su uso dónde y cuándo sea necesario y que además esté protegida de manera adecuada.

Para el control de la información documentada las empresas deberán hacer frente a las siguientes actividades: distribución, acceso, uso, recuperación, almacenamiento, conservación, control de cambios, retención y disposición. La información documentada de origen externo que se determine por la organización como necesaria para la planificación y operación del sistema de gestión, se identificará y controlará (Parrales, 2015).

Como nota, se reseña que el acceso a un documento puede implicar solamente la autorización para visualizar la información documentada o el permiso y la autoridad para verla y cambiarla (Quintero y Valencia, 2008).

La Dirección debe dotar a la organización de una estructura que permita cumplir con la misión y la visión establecidas. La implantación de la gestión de procesos se ha revelado como una de las herramientas de mejora de la gestión más efectivas para todos los tipos de organizaciones (González, 2009).

Cualquier actividad, o conjunto de actividades ligadas entre sí, que utiliza recursos y controles para transformar elementos de entrada (especificaciones, recursos, información, servicios,) en resultados (otras informaciones, servicios,) puede considerarse como un proceso. Los resultados de un proceso han de tener un valor añadido respecto a las entradas y pueden constituir directamente elementos de entrada del siguiente proceso.

Todas las actividades de la organización, desde la planificación de las compras hasta la atención de una 
reclamación, pueden y deben considerarse como procesos. Para operar de manera eficaz, las organizaciones tienen que identificar y gestionar numerosos procesos interrelacionados y que interactúan. La identificación y gestión sistemática de los procesos que se realizan en la organización y en particular las interacciones entre ellos, se conocen como enfoque basado en procesos (Severiche et al., 2016; Flores, 2012).

La gestión por procesos está dirigida a realizar procesos competitivos y capaces de reaccionar autónomamente a los cambios mediante el control constante de la capacidad de cada uno, la mejora continua, la flexibilidad estructural y la orientación de las actividades hacia la plena satisfacción del cliente y de sus necesidades. Es uno de los mecanismos más efectivos para que la organización alcance unos altos niveles de eficiencia (Mercado, 2014).

Para adoptar un enfoque basado en procesos, la organización debe identificar todas y cada una de las actividades que realiza. A la representación gráfica, ordenada y secuencial de todas las actividades o grupos de actividades se le llama mapa de procesos y sirve para tener una visión clara de las acciones que aportan valor al producto/servicio recibido finalmente por el cliente. En su elaboración debería intervenir toda la organización, a través de un equipo multidisciplinar con presencia de personas conocedoras de los diferentes procesos (Banguera, 2015).

Una característica importante de los procesos, que queda de manifiesto en cuanto se elabora el mapa de procesos, es que las actividades que lo constituyen no pueden ser ordenadas de una manera predeterminada, atendiendo a criterios sólo de jerarquía o de adscripción departamental.

Se puede decir que el proceso cruza transversalmente el organigrama de la organización y se orienta al resultado, alineando los objetivos de la organización con las necesidades y expectativas de los clientes, sin atender en sentido estricto a las relaciones funcionales clásicas (Holguín, 2012).

Las actividades de la organización son generalmente horizontales $y$ afectan a varios departamentos o funciones (comercial, tráfico, administración, etc.), como ilustra el siguiente gráfico. Esta concepción "horizontal" (actividades o procesos) se contrapone a la concepción tradicional de organización "vertical" (departamentos o funciones). Esto no significa que los procesos suplan o anulen las funciones. Como un pastel, se puede organizar por capas, pero se ha de servir por porciones.

La gestión de procesos consiste en dotar a la organización de una estructura de carácter horizontal siguiendo los procesos interfuncionales y con una clara visión de orientación al cliente final. Los procesos deben estar perfectamente definidos y documentados, señalando las responsabilidades de cada miembro, y deben tener un responsable y un equipo de personas asignado.

En este contexto es fundamental la figura del propietario, que es la persona que además de ocupar una determinada posición en el organigrama "convencional" (vertical), es responsable de analizar el proceso, mejorarlo y especialmente conseguir sus objetivos. La organización debe conocer quién es el propietario de cada uno de los procesos. El propietario asume la responsabilidad global de su gestión y de su mejora continua. Por ello, debe tener la suficiente autoridad para poder implantar los cambios en el proceso que él o el equipo de mejora estimen oportuno.

\section{Responsabilidad de la Dirección y Gestión de los Recursos}

En la norma ISO 9001 se habla del compromiso de la dirección donde afirma que la gestión de procesos consiste en dotar a la organización de una estructura de carácter horizontal siguiendo los procesos interfuncionales y con una clara visión de orientación al cliente final. Los procesos deben estar perfectamente definidos y documentados, señalando las responsabilidades de cada miembro, y deben tener un responsable y un equipo de personas asignado.

En este contexto es fundamental la figura del propietario, que es la persona que además de ocupar una determinada posición en el organigrama "convencional" (vertical), es responsable de analizar el 
proceso, mejorarlo y especialmente conseguir sus objetivos. La organización debe conocer quién es el propietario de cada uno de los procesos. El propietario asume la responsabilidad global de la gestión del proceso y de su mejora continua. Por ello, debe tener la suficiente autoridad para poder implantar los cambios en el proceso que él o el equipo de mejora del proceso estimen oportuno.

El liderazgo, compromiso y la participación activa de la alta gerencia son esenciales para desarrollar y mantener un sistema de gestión de la calidad eficaz y eficiente, que nos permita lograr beneficios para todas las partes interesadas. Es muy importante, por tanto que todo el personal perciba que la dirección está fuertemente comprometida con la implantación y mejora del sistema de gestión de calidad en la organización.

Cuando se habla de "proporcionar evidencia" se pueden diferenciar 2 aspectos:

Compromiso interno: ante el personal de la organización.

Compromiso externo: para la certificación.

La alta dirección debe establecer la política y asegurarse de que se establecen los objetivos, a la vez que debería definir métodos para medir el desempeño de la organización con el fin de determinar si se han alcanzado esos objetivos planificados.

La alta dirección también debe realizar las revisiones del sistema de gestión de calidad para evaluar la conveniencia, adecuación, eficacia y eficiencia del sistema, con respecto a los objetivos y a la política de calidad y debe llevarse a cabo con una frecuencia establecida en función de las necesidades de la organización.

\section{Determinación y revisión de los requisitos}

De los requisitos en la norma ISO 9001: 2015, se tiene el capítulo 8 operación, donde en el numeral 8.2. se habla de los requisitos para los productos y servicios, como lo son la comunicación con el cliente, que debe incluir el hecho de proporcionar la información relativa a los productos y servicios, la atención de las consultas, los contratos o los pedidos, incluyendo los cambios; obtener la retroalimentación de los clientes relativa a los productos y servicios, incluyendo las quejas de los clientes. Manipular o controlar las propiedades del cliente, establecer los requisitos específicos para las acciones de contingencia, cuando sea pertinente.

Determinación de los requisitos relacionados con los productos y servicios. Cuando determina los requisitos para los productos y servicios que se van a ofrecer a los clientes, la organización debe asegurarse de que: los requisitos para los productos y servicios se definen incluyendo cualquier requisito legal $y$ reglamentario aplicable: aquellos considerados necesarios por la organización, puede cumplir las declaraciones de los productos y servicios que ofrece.

Revisión de los requisitos relacionados con los productos y servicios. La organización debe asegurarse de que tiene la capacidad de cumplir los requisitos para los productos y servicios que se van a ofrecer a los clientes. La organización debe llevar a cabo una revisión antes de comprometerse a suministrar productos y servicios a un cliente, para incluir: los requisitos especificados por el cliente, incluyendo los requisitos para las actividades de entrega y las posteriores a la misma; los requisitos no establecidos por el cliente, pero necesarios para el uso especificado o para el uso previsto, cuando sea conocido; los requisitos especificados por la organización; los requisitos legales y reglamentarios adicionales aplicables a los productos y servicios; las diferencias existentes entre los requisitos de contrato - pedido y los expresados previamente. La organización debe asegurarse de que se resuelven las diferencias existentes entre los requisitos del contrato - pedido y los expresados previamente. La organización debe confirmar los requisitos del cliente antes de la aceptación, cuando el cliente no proporcione una declaración documentada de sus requisitos.

Cambios en los requisitos para los productos y servicios. La organización debe asegurarse que cuando se cambien los requisitos para los productos y servicios, la información documentada pertinente sea 
modificada y que las personas correspondientes sean conscientes de los requisitos modificados.

\section{Medición, Análisis y Mejora}

La empresa tiene que establecer: Que necesita realizar un seguimiento y una medición. Los métodos de seguimiento, medición, análisis y evaluación necesarios para conseguir resultados válidos. Cuando se tienen que realizar los seguimientos $y$ las mediciones. Cuando se deben analizar y evaluar los resultados de seguimiento y medición. La empresa tiene que realizar una evaluación del desempeño y la eficiencia del Sistema de Gestión de la Calidad. La empresa tiene que conservar toda la información documentada que crea necesaria para que le sirva de evidencia.

Satisfacción del cliente. La empresa tiene que realizar el seguimiento de las percepciones de los clientes del grado en el que se cumplen todas las necesidades y las expectativas. La empresa tiene que determinar los métodos para conseguir, realizar el seguimiento y revisar la información.

Análisis y evaluación. La empresa tiene que analizar y realizar una evaluación de los datos y la información apropiada que surge de realizar el seguimiento y la medición. Los resultados del análisis tienen que utilizarse para realizar la evaluación: La conformidad de los productos y los servicios. El nive de satisfacción de los clientes. El desempeño y la eficiencia del SGC. Si lo que se ha planteado se ha implantado con eficacia. La eficiencia de todas las acciones tomadas para realizar los riesgos y las oportunidades. La labor que realizan los proveedores externos. La necesidad de mejorar el Sistema de Gestión de la Calidad.

Se debe determinar que es necesario seguir, medir, analizar y evaluar todos los métodos que se emplean y se deben emplear en las actividades. En la norma no se especifica mediante que método se obtiene la información, ya que no es un requisito obligatorio. Existe una parte de la norma en la que se enumeran una serie de elementos que la empresa tiene que analizar y evaluar, los resultados pueden resultar muy útiles para ser revisados por la dirección. En conclusión, la organización decide lo que debe evaluar para determinar la eficacia del Sistema de Gestión de Calidad. De la misma forma, se tiene que asegurar la satisfacción del cliente y la eficiencia de los procesos y las operaciones.

\section{Conclusiones}

El presente informe consta de una revisión de la literatura científica desarrollada sobre la temática de los sistemas de gestión de calidad y los sistemas integrados de gestión, que nacen como solución ante la necesidad de integrar los parámetros de normas que tienen correspondencia técnica y estructural para garantizar la calidad en las empresas a través del correcto desarrollo de procesos, recursos y obtención de resultados de alto valor para los clientes.

Se puede concluir que los sistemas integrados de gestión de la calidad, equivalen a una optimización de los recursos requeridos para la garantía de la calidad total en una compañía, así, por medio de la integración de los estándares que regulan la calidad, el medio ambiente y la seguridad y salud ocupacional, se logra hacer una correcta administración de cada una de las potencialidades de la empresa, para garantizar su competitividad en el mercado.

Durante la revisión se encontraron diferentes beneficios para las empresas que desarrollen este tipo de sistemas, como es el caso de que con una sola auditoria se podría evaluar la aplicación de los tres estándares referenciados a lo largo de este trabajo, además, este tipo de sistemas garantiza a las empresas la eliminación de tareas duplicadas, además del gasto innecesario de diferentes recursos que pueden llegar a optimizarse.

\section{Referencias}

Aguilera, L. (2012). Los sistemas integrados de calidad y ambiente: gestión ambiental, ciencia, tecnología y El equidad social en la educación universitaria. Innovación Educativa.

Amparo, N. (2009). Diseño del sistema de gestión en seguridad y salud ocupacional, bajo los requisitos de 
la norma NTC OHSAS18001 en el proceso de fabricación de cosméticos para la empresa Wilcos S.A [Trabajo previo al título de Ingeniero Industrial]. Bogotá: Pontificia Universidad Javeriana.

Banguera, L. (2015). Propuesta para la implementación de un modelo de sistema integrado de gestión en calidad. Ecuador: Universidad de Guayaquil.

Beltrán, J., Carmona, M., \& Carrasco, R. (2009). Guía para una gestión basada en procesos. Instituto Andaluz de tecnología.

Bedoya, E., Carrillo, M., Severiche, C. (2017). La mejora continua. enfoque moderno hacia la medición de la calidad en universidades. Journal Industrial Neo Of Technologies. v. 4 fasc. 1 p. $5-13$.

Benavides, G. (2013). Ética empresarial y sistemas integrados de gestión: organizaciones pioneras certificadas en Bogotá. SIGNOS, 71-86.

Bestraten, M. (2000). NTP 576: Integración de sistemas de gestión: prevención de riesgos laborales, calidad y medio ambiente. España: Ministerio de trabajo y asuntos sociales.

Cachay, G. (2009). Implementación de un sistema integrado de gestión en la empresa Paraíso. Lima.

Cárdenas, C. (2016). Diseño de un sistema integrado de gestión basado en las normas ISO para la empresa La Casa del Ingeniero. Bogotá: Escuela Colombiana de Ingeniería Julio Garavito.

Cortés, a. (2014). Sistemas integrados de gestión y responsabilidad social en empresas agroindustriales de los departamentos de Cauca y Valle del Cauca, Colombia. Informador Técnico.

Damaso, T. (2010). Integración de sistemas de gestión ambiental, seguridad y salud en el trabajo. Gestión ambiental, Seguridad y salud ocupacional.

De Velasco, J. (2009). Gestión por procesos. Esic Editorial.

Del prado, M. (2016). Propuesta de un modelo de sistema integrado de gestión de la información documental para las organizaciones. Ediciones complutense.

Duque, D. (2010). Modelo teórico para un sistema integrado de gestión (seguridad, calidad y ambiente). Actualidad y nuevas tendencias, 115-130.

Fernández, L. (2014). Diseño de un sistema integral de gestión de la calidad, medio ambiente y riesgos laborales. Valencia.

Flores, A. (2013). Efectos de la implementación del sistema integrado de gestión en los grupos de interés de las empresas industriales, comerciales y de servicios del área metropolitana del Valle de Aburrá. Universidad de Manizales.

Flores, G. (2012). Guía metodológica para la implementación de un sistema integrado de gestión en le empresa Cortiplast s.a.

Fraguela, J., Carral, L., \& Iglesias, G. (2011). La integración de los sistemas de gestión. Necesidad de una nueva cultura empresarial. Dyna, 44-49.

García, C., \& Espinel, J. (2016). Diseño de un sistema de gestión de calidad basado en los requisitos de las NTC ISO 9001: 2015 para el proceso curricular de administración ambiental de la universidad distrital Francisco José de Caldas con fines de acreditación. Bogotá: Universidad Distrital Francisco José de Caldas.

Gisbert, V., \& Esenglediev, R. (2015). Sistemas integrados de gestión y los beneficios. 3c Empresa, 246-257.

González, A. (2008). Diseño de un sistema de gestión de la calidad con un enfoque de ingeniería de la calidad. Ingeniería industrial, 1-6.

González, H. (2013). Enfoque basado en procesos como principio de la gestión. 3c Empresarial, 120-131.

González, N. (2009). Diseño del sistema de gestión en seguridad y salud ocupacional, bajo los requisitos de la norma NTC OHSAS18001 en el proceso de fabricación de cosméticos.

González, S. (2011). Sistemas integrados de gestión, un reto para las pequeñas y medianas empresas. Escenarios, 69-89.

Hernández, H., Martínez, D., \& Cardona, D. (2015). Enfoque basado en procesos como estrategia de dirección para las empresas de transformación. Saber, Ciencia y Libertad, 141-150.

Hernández, S. (2015). Ventaja competitiva de los sistemas de gestión integrados de la calidad, enfocados en la responsabilidad social empresarial como valor agregado. Bogotá: Universidad Militar Nueva Granada.

Herrera, K. (2014). Sistemas integrados de gestión HSEQ, más que un requisito exigible [artículo de reflexión cofín de grado]. Cartagena: Universidad San Buenaventura.

Holguín, G. (2012). Implantación de un sistema integrado de gestión en calidad, medio ambiente, seguridad y salud en el trabajo responsabilidad social corporativa en el laboratorio bioplex.

Leung, M. (2010). Diseño de un sistema integrado de gestión para la empresa C.I Recycables de la ciudad de Cartagena. Cartagena: Universidad Tecnológica de Bolívar.

López, a. (2008). Sistema integrado de gestión para empresas turísticas. Universidad de Salamanca.

Luero, J. (2017). Propuesta de alineación del sistema 
integrado de gestión del ICBF Regional Bogotá con las normas ISO 9001:2015, ISO 14001: 2015 Y OHSAS 18001: 2007. SIGNOS, 57-72.

Marín, R. (2013). El rol de la gestión del conocimiento en la implementación de un sistema integrado de gestión. LOGOS, CIENCIA Y TECNOLOGÍA.

Matiz, V., \& Ramírez, G. (2011). Diseño de un sistema integrado de gestión en la empresa Pintumarca Núñez y Nogales Cía. Ltda. Pontificia Universidad Javeriana.

Medina, D. (2012). Enfoque basado en proceso. Oficina Asesora de Planeación.

Mercado, C. (2014). Diseño de un sistema de gestión integral gerencial bajo las NTC ISO9001:2008, ISO 14001 y OHSAS 18001 aplicado a la empresa Latco Solitions SAS.

Morales, O. (2017). Procedimiento para la documentación de los procesos en los sistemas de gestión de la calidad de la ciencia y la técnica universitaria. Retos de la dirección, 111-135.

Parrales, Y. (2015). Implementación de un sistema integrado de gestión de la calidad, ambiente y seguridad para una planta dedicada a la elaboración de alimentos. Escuela Politécnica del Litoral.

Paulí, E., \& Carretero, J. (2009). Sistemas integrados de gestión de calidad y medio ambiente: evidencia empírica en la industria española. Universidad de les Illes Balears.

Peña, D. (2011). Sistemas integrados de gestión de la calidad, el medio ambiente, la seguridad y salud en el trabajo, según los enfoques normalizados. Ciencias Holguín, 1-11.

Pérez, E. (2010). Sistema de gestión integral bajo la norma ISO 9001, ISO 4001 y OHSAS 18001 en una cooperativa. Universidad Cooperativa de Colombia.

Petro, G. (2013). Niveles de responsabilidad y autoridad del sistema integrado de gestión. Bogotá: Alcaldía de Bogotá.

Ponce, G. (2015). Diseño del sistema integrado de gestión de la calidad, seguridad salud ocupacional y ambiental de acuerdo a las normas ISO 9001: 2008, OHSAS 18001: 2007, ISO 14001: 2004 para la empresa Siembranueva S.A. Guayaquil: Universidad Politécnica Salesiana.

Pulles, R., \& Bataller, M. (2016). Modelo de sistema integrado de gestión para una dirección de investigación medioambiental de biocubafarma. Revista Cenic, 6-16.

Quintero, E., \& Valencia, S. (2008). Diagnóstico para la implementación de un sistema de gestión de calidad ISO 9001:2000 al interior de la empresa Maderas
Yumbo. Bogotá.

Rodríguez, G., Lezcano, D., \& Varela, Y. (2010). El enfoque de procesos como principio básico de los sistemas de gestión de la calidad. Revista científica Avances, 120135.

Rubio, J. (2002). Gestión de la prevención de riesgos laborales. 1a edición. Editorial Díaz de Santos.

Severiche, C., Muñoz, D. y Jaimes, J. (2016). Gestión del conocimiento en sector de agua potable y saneamiento básico en Colombia. Revista Omnia, 22(1), 91-105.

Tejada, V. (2006). Modelo de un sistema integrado de gestión para la subdirección redes de transmisión energía enfocado en las normas ISO 9001, ISO 14001 y OHSAS 18001. Medellín: Universidad de Antioquia.

Tejada, V. H. (2006). Modelo de un sistema integrado de gestión para la subdirección redes de transmisión energía enfocado en las normas ISO 9001, ISO 14001 y OHSAS 18001 [Trabajo de grado previo al título de Especialista en Asesoría y Consultoría de Organizaciones]. Medellín: Universidad de Antioquia.

Vidal, E., \& Soto, E. (2013). Implantación de los sistemas integrados de gestión. Tourism \& Management Studies, 4, 1112-1221. 